OPEN ACCESS

Check for updates

\section{Use of metformin to prolong gestation in preterm pre-eclampsia: randomised, double blind, placebo controlled trial}

\author{
Catherine A Cluver, ${ }^{1,2,3}$ Richard Hiscock, ${ }^{2}$ Eric H Decloedt,, ${ }^{4}$ David R Hall, ${ }^{1}$ Sonja Schell, ${ }^{1}$ \\ Ben W Mol, ${ }^{5}$ Fiona Brownfoot, ${ }^{2,3}$ Tu'uhevaha J Kaitu'u-Lino, ${ }^{2,3}$ Susan P Walker, 2,3 \\ Stephen Tong ${ }^{2,3}$
}

${ }^{1}$ Department of Obstetrics and

Gynaecology, Stellenbosch University, Tygerberg Hospital, 7505, Cape Town, South Africa Department of Obstetrics and Gynaecology, University of Melbourne, Victoria, Australia

${ }^{3}$ Mercy Perinatal, Mercy Hospital for Women, Heidelberg, Victoria, Australia

${ }^{4}$ Department of Medicine, Division of Clinical

Pharmacology, Stellenbosch

University, Tygerberg Hospital, Cape Town, South Africa

${ }^{5}$ Department of Obstetrics and Gynaecology, Monash School

of Medicine, Monash University, Melbourne, Victoria, Australia

Correspondence to: C A Cluver cathycluver@sun.ac.za

(or @cathycluver on Twitter

ORCID 0000-0002-0406-8964)

Additional material is published online only. To view please visit the journal online.

Cite this as: BMJ 2021;374:n2103 http://dx.doi.org/10.1136/bmj.n2103

Accepted: 23 August 2021
${ }^{2}$ Translational Obstetrics Group,

\section{ABSTRACT}

OBJECTIVE

To evaluate whether extended release metformin expectantly managed for preterm pre-eclampsia.

\section{DESIGN}

Randomised, double blind, placebo controlled trial.

SETTING

Referral hospital in Cape Town, South Africa.

\section{PARTICIPANTS}

180 women with preterm pre-eclampsia between $26+0$ to $31+6$ weeks' gestation undergoing expectant management: 90 were randomised to extended release metformin and 90 to placebo.

\section{INTERVENTION}

$3 \mathrm{~g}$ of oral extended release metformin or placebo daily, in divided doses, until delivery.

\section{MAIN OUTCOME MEASURE}

The primary outcome was prolongation of gestation.

RESULTS

Of 180 participants, one woman delivered before taking any trial drug. The median time from randomisation to delivery was 17.7 days (interquartile range 5.4-29.4 days; $n=89$ ) in the metformin arm and $10.1(3.7-24.1 ; n=90)$ days in the placebo arm, a median difference of 7.6 days (geometric mean ratio $1.39,95 \%$ confidence interval 0.99 to $1.95 ; \mathrm{P}=0.057$ ). Among those who continued to take the trial drug at any dose, the median prolongation of gestation in the metformin arm was 17.5 (interquartile range 5.4-28.7; $n=76$ ) days compared with 7.9 (3.0-22.2; $\mathrm{n}=74$ ) days in the placebo arm, a median difference of 9.6 days (geometric mean ratio 1.67, $95 \%$ confidence interval 1.16 to 2.42 ). Among those who took the full dosage, the median prolongation of gestation in the could be used to prolong gestation in women being

\section{WHAT IS ALREADY KNOWN ON THIS TOPIC}

Few randomised trials have been done of drugs that aim to slow disease progression of preterm pre-eclampsia and none have clearly found success

Preclinical studies have identified metformin as a potential treatment for preeclampsia as its actions might mitigate placental and maternal vessel disease and in animal models it has been shown to rescue a pre-eclampsia phenotype Metformin to treat pre-eclampsia has not been evaluated in trials

\section{WHAT THIS STUDY ADDS}

Extended release metformin in women with preterm pre-eclampsia (26-32 weeks' gestation) prolonged pregnancy gestation by a week

Metformin might have resulted in shorter hospital admission in the neonatal nursery after birth metformin arm was 16.3 (interquartile range 4.8-28.8; $\mathrm{n}=40)$ days compared with $4.8(2.5-15.4 ; \mathrm{n}=61)$ days in the placebo arm, a median difference of 11.5 days (geometric mean ratio $1.85,95 \%$ confidence interval 1.14 to 2.88). Composite maternal, fetal, and neonatal outcomes and circulating concentrations of soluble fms-like tyrosine kinase-1, placental growth factor, and soluble endoglin did not differ. In the metformin arm, birth weight increased non-significantly and length of stay decreased in the neonatal nursery. No serious adverse events related to trial drugs were observed, although diarrhoea was more common in the metformin arm.

\section{CONCLUSIONS}

This trial suggests that extended release metformin can prolong gestation in women with preterm preeclampsia, although further trials are needed. It provides proof of concept that treatment of preterm pre-eclampsia is possible.

TRIAL REGISTRATION

Pan African Clinical Trial Registry

PACTR201608001752102 https://pactr.samrc.ac.za/.

\section{Introduction}

Pre-eclampsia is a leading cause of maternal and neonatal morbidity and mortality. ${ }^{12}$ In South Africa, hypertensive disorders of pregnancy account for $15 \%$ of maternal deaths. ${ }^{3}$ Preterm pre-eclampsia is one of the most severe variants, affecting $0.5 \%$ of all pregnancies (ie, $10 \%$ of all women with pre-eclampsia). ${ }^{4}$ Preterm pre-eclampsia is also associated with substantially more maternal and neonatal morbidity and mortality compared with pre-eclampsia occurring at term gestation. ${ }^{45}$ Apart from delivery, no treatment exists for pre-eclampsia.

Preterm pre-eclampsia often leads to delivery for maternal indications, which results in iatrogenic premature delivery and places the neonate at risk of major neonatal disability and death, particularly in low and middle income countries. If a safe treatment was available that delayed the progression of preterm pre-eclampsia, babies could be delivered at a less preterm gestation. Reducing the neonatal burden of complications from severe prematurity could confer important benefits. Efforts to find drugs that are safe to administer during pregnancy and can slow the progression of preterm pre-eclampsia have been unsuccessful. ${ }^{6-10}$

Preclinical laboratory studies have identified the oral blood glucose lowering drug metformin as a potential candidate for the treatment of pre-eclampsia. ${ }^{11-13} \mathrm{We}$ evaluated whether extended release metformin can 
prolong gestation among women with a diagnosis of preterm pre-eclampsia.

\section{Methods}

Pharmacokinetics study

To assess the pharmacokinetics of extended release metformin in women with preterm pre-eclampsia, we performed an open label study of 15 women with preterm pre-eclampsia before the trial started (see supplementary file material and figure A for details).

\section{Trial design and participants}

We performed a double blind, placebo controlled clinical trial to compare prolongation of gestation among women with a diagnosis of preterm preeclampsia at $26+0$ to $31+6$ weeks' gestation. The women were treated with either $3 \mathrm{~g}$ of extended release metformin ( $1 \mathrm{~g}$ three times daily) or matching placebo. The trial site was Tygerberg Hospital in Cape Town, South Africa; a large academic referral centre situated in a region with high rates of pre-eclampsia.

We recruited women with a diagnosis of preterm pre-eclampsia at $26+0$ to $31+6$ weeks' gestation. Only those who were candidates for expectant management and had no clinical indication for immediate delivery, based on the clinical assessments of the attending doctors, were invited to participate. Eligible women were those who were able to give informed consent, were not currently using metformin, and had a fetus without structural anomalies.

Exclusion criteria included established maternal or fetal compromise that necessitated immediate delivery, diabetes or gestational diabetes, contraindications to metformin (baseline creatinine $>124 \mu \mathrm{mol} / \mathrm{L}$, hypersensitivity to metformin, or a metabolic acidosis), use of drugs that might interact with metformin (glyburide, furosemide, or cationic drugs), and multiple gestations. Pre-eclampsia was defined according to the International Society for the Study of Hypertension in Pregnancy Classification ${ }^{14}$, but we only included those who had important proteinuria ( $>300 \mathrm{mg}$ of protein measured on a 24 hour urine collection). Women with either new onset hypertension after 20 weeks' gestation or women receiving treatment for chronic hypertension, with loss of blood pressure control were invited to participate provided they also fulfilled our definition of pre-eclampsia.

Expectant management involved hospital admission with close maternal and fetal surveillance. Maternal surveillance included blood pressure measurements every four hours, twice daily clinical assessments, daily urinalysis, and twice weekly biochemical testing. Biochemical tests measured haemoglobin, urea, and creatinine levels and platelet count. If biochemical abnormalities were detected, then further investigations were done, including assessing levels of liver enzymes and lactate dehydrogenase. Fetal surveillance included ultrasonography for fetal size and Doppler velocimetry soon after admission, followed by reviews twice a week or more, as indicated (such as the presence of absent end diastolic flow in the umbilical artery). Cardiotocography was performed every six hours. To enhance fetal lung maturity, two doses of betamethasone 24 hours apart were given to participants when first admitted, followed by a single repeat dose one week later if no delivery had occurred, according to hospital protocol. ${ }^{15}$

Delivery before 34 weeks' gestation was a clinical decision made by the woman's treating team. Of the authors, only DRH was involved in clinical decisions for some participants. At Tygerberg hospital, expectant management is stopped at 34 weeks' gestation and delivery takes place. Owing to the high demand for neonatal beds at Tygerberg hospital, neonates are transferred to a step-down facility (primary or secondary level hospital), or home as soon as allowed according to the neonate's condition. This is an individualised clinical decision based on the neonate's weight, gestation at delivery, feeding schedule, and progress.

This trial was prospectively registered with the Pan African Clinical Trial Registry on 29 August 2016. The trial protocol has been published. ${ }^{16}$ The study was conducted with fidelity to the protocol, which is available along with the prespecified statistical analysis plan (see supplementary material). All participants provided written informed consent. Study data were collected and managed using REDCap electronic data capture tools. ${ }^{17}$

\section{Randomisation and trial group assignment}

The women were randomly assigned to receive extended release metformin or placebo using a 1:1 online, web based sequence generator. Because gestation might affect the length of pregnancy prolongation, we stratified randomisation according to gestation at recruitment (stratum 1: $26+0$ to $\leq 28+6$ weeks; stratum 2: $29+0$ to $31+6$ weeks) using blocks of size four to six. The South Australian Health and Medical Research Institute in Adelaide, Australia (https://www.sahmri. org/) set up the randomisation. CAC and SS selected and randomised the participants.

Both the metformin and the placebo tablets were manufactured by Merck Healthcare (Darmstadt, Germany) and provided as a research donation. Each metformin tablet contained $500 \mathrm{mg}$ of metformin hydrochloride. The placebo tablets contained no active ingredient. The tablets were identical in size, thickness, physical properties, and appearance. The trial pharmacy at TREAD Research, Tygerberg Hospital (https://www.tread.co.za/) manufactured identical packages, each labelled with unique identifiers, according to a randomly generated sequence known only to the randomisation centre. If follow-up packs were required, the randomisation centre dispatched packs from the same allocation. Trial participants, doctors, care providers, and investigators had no access to the randomisation list, and allocation concealment was maintained throughout the trial.

The women initially received one trial tablet three times a day, with the dose increased to two tablets three times a day as tolerated. If side effects developed, the 
dose could be decreased to one tablet twice a day and then increased if symptoms improved, to a maximum of $3000 \mathrm{mg} /$ day. We recommended that treatment should be taken from randomisation until delivery. After delivery, the treatment packs were collected and the remaining tablets counted by CAC and SS. The trial midwives reviewed participants daily for drug compliance and adverse events. Serious adverse events were reported to both the data monitoring and safety committee and the health research ethics committee and were handled in accordance with good clinical practice guidelines.

Plasma samples were collected from the women at randomisation and twice a week until birth. The samples were processed and frozen at $-80^{\circ} \mathrm{C}$. After recruitment was complete, the frozen samples were shipped in one batch to Melbourne, Australia where ST, TJK, and FB measured levels of circulating soluble fms-like tyrosine kinase-1, placental growth factor, and soluble endoglin. (See supplementary material for details on how samples were collected, assayed, and reported.)

\section{Outcome measures}

The primary outcome was prolongation of gestation, measured from time of the first dose of trial drug until delivery in days and hours. We prespecified an analysis for treatment received (women who took the trial drugs at any dose) and a further analysis limited to women who took the full dosage (ie, from randomisation to delivery). Secondary outcomes included composite maternal, fetal, or neonatal outcomes and circulating levels of anti-angiogenic biomarkers associated with pre-eclampsia. The maternal composite outcome included any of the following: maternal death, eclampsia, pulmonary oedema (oxygen saturation $\leq 90 \%$, with clinical symptoms requiring treatment), severe renal impairment (serum creatinine level $\geq 125$ $\mu \mathrm{mol} / \mathrm{L})$, a cerebral vascular event, placental abruption, and liver haematoma or rupture. The fetal composite outcome included major changes in heart rate patterns on the non-stress test or cardiotocograph, as defined by the attending clinician, that necessitated delivery; intrauterine fetal death; or fetal growth restriction at birth, defined as an estimated fetal weight less than the third centile on the GROW (gestation related optimal weight) chart or less than the third centile on the World Health Organization charts, or both. ${ }^{18} 19$ The neonatal composite outcome included any of the following: neonatal death within six weeks of the expected due date, grade III or IV intraventricular haemorrhage (on cranial ultrasonography), necrotising enterocolitis (on radiographic studies), or bronchopulmonary dysplasia (needing oxygen at 28 days of life). Exploratory outcomes included individual clinical outcomes (listed in table 2).

\section{Statistical analysis}

The sample size was initially informed by a retrospective cohort study from our unit, where the mean prolongation for expectant management of preterm pre-eclampsia was 11 days (SD 7 days) and a coefficient of variation of $0.64 .{ }^{20}$ A normal distribution was assumed, and the sample size was uplifted by $15 \%$ to account for non-normality, resulting in a sample size of 120 women. In 2018 we completed the Pre-eclampsia Intervention Trial (evaluating esomeprazole), which was run at the same site and recruited a similar population of participants. ${ }^{9}$ The overall prolongation in the placebo arm of the Preeclampsia Intervention Trial was 13.1 (SD 12.2) days. We therefore recalculated the sample size for the current trial, and settings included a coefficient of variation set higher, at 1.0 , a power of $90 \%$, and a detectable geometric mean ratio of 1.6. Overall, 130 participants were required (65 in each group) for a power of $90 \%$ to detect a prolongation of gestation longer than five days, significant at a two sided 5\% level. We increased our recruitment target to 75 women in each group to compensate for anticipated dropouts and amended the protocol (version dated 26 January 2018). ${ }^{15}$ During the trial, the incidence of side effects was higher than anticipated, resulting in a higher than expected number of participants who decreased or stopped the trial drug. While still blinded, the investigators made a request to the data monitoring and safety committee on 19 June 2019 to increase the sample size to 90 women in each arm, and this was approved. The final target recruitment was therefore 180 participants.

We prepared a prespecified statistical analysis plan that was finalised before unblinding (see supplementary material). Personal and clinical data are presented as means (standard deviations), medians (interquartile ranges), minimums, maximums, and numbers (percentages), according to type and distribution. The primary outcome analysis was performed using ordinary least squares regression of log transformed prolongation. Bootstrap resampling (10000 replications) was used to estimate the associated 95\% confidence intervals. The use of geometric mean ratio followed input from the statistical reviewer for The BMJ during peer review, who highlighted the inadequate sample size if quantile median regression was used. The use of geometric mean ratio is concordant with the prespecified sample size and power calculations. Gestational strata are presented as covariates, and the results are presented as geometric mean ratios with corresponding 95\% confidence intervals and $\mathrm{P}$ values.

We performed supplementary survival analyses using Cox proportional hazards regression adjusted for strata of gestational age and presented as hazard ratios (95\% confidence intervals), along with a KaplanMeier survival curve to illustrate the prolongation of gestation. The prespecified composite maternal and neonatal outcomes were tested using an exact logistic regression model, with group and strata as covariates. We considered a two sided $\mathrm{P}$ value $<0.05$ to be significant. For the exploratory outcomes, we used the same statistical techniques as those used to compare the primary and secondary outcomes, but without correction for strata. Software Stata v16 (Stata Statistical Software Release 16; Stata) was used. 


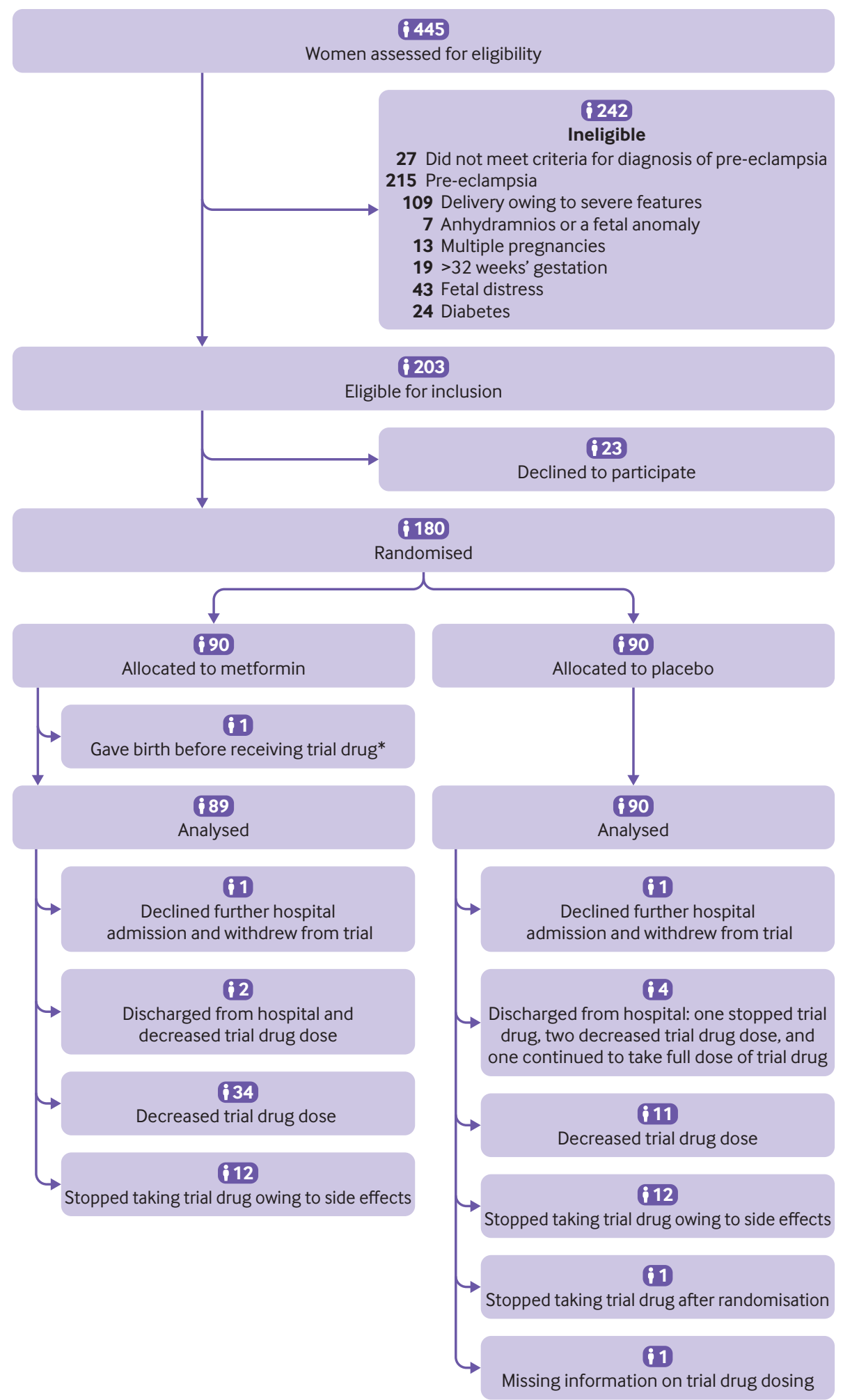

Fig 1 | Flowchart of screening, randomisation, and follow-up. *Primary outcome was measured from time of taking first tablet until delivery. This woman delivered before taking her first dose so it was not possible to calculate prolongation of gestation

\section{Patient and public involvement}

Although we did not actively seek patient or public involvement in the development of the protocol, the steering committee of international content experts included a woman who had experienced preeclampsia. Furthermore, we spoke to patients about the study and asked a member of the public to read our manuscript before submission. Participants are acknowledged and thanked for their contribution and participation. A dissemination strategy has been developed to work closely with stakeholders and knowledge users to facilitate transfer of the findings 
to relevant end users, including patients, patient organisations, and clinicians.

\section{Results}

Women were recruited to the trial between February 2018 and March 2020 after the pharmacokinetic study was completed (see supplementary material). Of the 203 women who fulfilled the inclusion criteria, 23 (11\%) declined to participate, mostly because they did not want to take more tablets or they were not willing to commit to inpatient care until 34 weeks' gestation. In total, 180 women were randomly allocated: 90 to extended release metformin and 90 to placebo. After randomisation, one woman in the metformin arm delivered before taking a first dose of the trial drug. As the primary outcome was measured from time of taking the first dose to delivery, the prolongation of gestation in this woman could not be calculated and she was therefore excluded, leaving 179 women to be included in the analysis. Two women in the metformin arm and four in the placebo arm were discharged home as the treating clinicians decided after randomisation that they did not meet the criteria for a diagnosis of preeclampsia. One woman in the metformin arm and one in the placebo arm declined further hospital admission and withdrew before delivery (fig 1).

Table 1 shows the baseline characteristics of the women. The median gestational age at recruitment was 29 weeks in both the metformin and the placebo arms, with $40 \%$ of women recruited before these gestations (stratum 1). More women in the metformin arm than placebo arm were using aspirin (11\% v 4\%) and calcium supplements (13\% v9\%). At randomisation, almost half of the fetuses had an estimated weight (determined using ultrasonography and referenced against fetal charts) <10th centile: $47 \%$ using the GROW reference chart(18) or $48 \%$ using WHO reference charts(19)). Supplementary figure B shows baseline soluble fms-like tyrosine kinase- 1 to placental growth factor ratios.

\section{Primary outcome}

The median time from randomisation to delivery in the metformin arm was 17.7 days (interquartile range 5.4-29.4 days; $n=89$ ) and in the placebo arm was 10.1 (3.7-24.1; $n=90)$ days, a median difference of 7.6 days (geometric mean ratio 1.39, 95\% confidence interval 0.99 to $1.95, \mathrm{P}=0.057$ (table 2). No evidence of an interaction between gestational age strata and treatment effect was found $(\mathrm{P}=0.72)$. Figure 2 shows the Kaplan-Meier survival curve for prolongation of gestation in days between the treatment arms overall and by gestational age stratum.

A prespecified analysis compared prolongation of gestation among participants who continued to take the trial drug, at any dose. Thirteen participants in the metformin arm and 15 in the placebo arm stopped treatment before delivery and, along with one participant in the placebo arm with incomplete dosing information, were not included in this analysis. Thirty six women in the metformin arm and 13 in the placebo arm decreased the number of tablets taken. The median time for prolongation of gestation in the metformin arm was 17.5 days (interquartile range 5.4-28.7 days; $\mathrm{n}=76)$ and in the placebo arm was 7.9 days (3.0-22.2; $n=74)$, a median difference of 9.6 days (geometric mean ratio $1.67,95 \%$ confidence interval 1.15 to 2.42) (supplementary table A).

A further prespecified analysis compared the prolongation of gestation in women who took the full dosage of trial drug (that is, from randomisation to delivery). In these women, the median time from randomisation to delivery in the metformin arm was 16.3 (interquartile range 4.8-28.8; $\mathrm{n}=40$ ) days and in the placebo arm was $4.8(2.5-15.4 ; n=61)$ days, a median difference of 11.5 days (geometric mean ratio $1.85,95 \%$ confidence interval 1.16 to 2.96 ) (supplementary table A).

As more women in the metformin arm than placebo arm took aspirin and calcium supplements during pregnancy, a sensitivity analysis was performed adjusted both individually and jointly for aspirin, calcium, and HIV status (supplementary table B). A prespecified sensitivity analysis was also done for fetal growth restriction at inclusion. The magnitude of the point estimate for geometric mean ratio, interpreted as percentage change, was stable individually and jointly (ranging from 1.36 to 1.40), confirming no interaction.

\section{Secondary outcomes}

The metformin and placebo arms did not differ for the prespecified secondary composite endpoints for maternal ( $6 \%$ v 4\%; difference $1.3 \%$, 95\% confidence interval $-5.2 \%$ to $7.6 \%)$, fetal $(66 \% \vee 70 \% ;-4.2 \%$, $-17.8 \%$ to $9.5 \%)$, or neonatal outcomes (16\% v 18\%; $-2.0 \%,-13.0 \%$ to $8.9 \%$ ) (table 2). Circulating levels of soluble fms-like tyrosine kinase-1, placental growth factor, or soluble endoglin concentrations, or the soluble fms-like tyrosine kinase-1 to placental growth factor ratio did not differ (supplementary figures $\mathrm{C}$ and $\mathrm{D}$ ).

\section{Exploratory outcomes}

More women in the metformin arm than placebo arm reached 34 weeks' gestation $(40 \% v 28 \% ; 12.7 \%$, $-1.1 \%$ to $26.4 \%$ ) and fewer women in the metformin arm delivered because of fetal indications (33\% v 44\%; $-11.9 \%,-26.0 \%$ to $2.3 \%$ ); but these results were not statistically significant (table 2). In the metformin arm, birth weights were non-significantly increased (1620 g v 1510 g; difference 110 g, 95\% confidence interval -80 to 300) and median length of admission in the Tygerberg Hospital neonatal nursery was nonsignificantly shorter (11 days $v 16$ days, a difference of 5 days, geometric mean ratio $0.86,95 \%$ confidence interval 0.62 to 1.20). On completion of the trial, we obtained access to an electronic system (Single Patient Viewer https://spv.jembi.org/) that tracks the total length of stay in any neonatal nursery (that is, the total period of admission at Tygerberg Hospital (level 3 neonatal nursery) plus the period of admission if the neonate was transferred to a neonatal nursery unit at another hospital). The investigator who collected these 


\begin{tabular}{|c|c|c|}
\hline Characteristics & Metformin arm $(n=89)$ & Placebo arm $(n=90)$ \\
\hline Median (interquartile range) gestation at randomisation (weeks+days) & $29+3(28+2-30+6)$ & $29+5(28+0-31+0)$ \\
\hline Gestation <29 weeks ( +0 days) at randomisation & $36(40)$ & $36(40)$ \\
\hline Median (interquartile range) maternal age (years) & $29(25-33)$ & $27(23-34)$ \\
\hline Median (interquartile range) body mass index & $30(26-36)$ & $29(25-35)$ \\
\hline \multicolumn{3}{|l|}{ Ethnicity: } \\
\hline Black & $59(66)$ & $61(68)$ \\
\hline Mixed & $29(33)$ & $29(32)$ \\
\hline White & $1(1)$ & 0 \\
\hline Current smoker & $13(15)$ & $15(17)$ \\
\hline Aspirin use during pregnancy & $10(11)$ & $4(4)$ \\
\hline Calcium supplement use during pregnancy & $12(13)$ & $8(9)$ \\
\hline HIV positive & $18(20)$ & $20(22)$ \\
\hline Chronic hypertension & $24(27)$ & $22(24)$ \\
\hline \multicolumn{3}{|l|}{ Parity: } \\
\hline Nulliparous & $27(30)$ & $31(34)$ \\
\hline Multiparous & $62(70)$ & $59(66)$ \\
\hline \multicolumn{3}{|l|}{ Hypertension in previous pregnancy: } \\
\hline No & $27(44)$ & $26(44)$ \\
\hline Yes & $33(53)$ & $31(53)$ \\
\hline New paternity in current pregnancy & $18(20)$ & $16(18)$ \\
\hline \multicolumn{3}{|l|}{ Mean (SD) highest blood pressure before randomisation $(\mathrm{mm} \mathrm{Hg})$ : } \\
\hline Systolic & $167(18.1)$ & $169(16.0)$ \\
\hline Diastolic & $100(12.9)$ & $102(14.1)$ \\
\hline Mean (SD) haemoglobin (g/L) & $116(14.7)$ & $116(13.0)$ \\
\hline Mean (SD) platelet count $\left(\times 10^{9} / \mathrm{L}\right)$ & $234(82.5)$ & $231(74.9)$ \\
\hline Mean (SD) urea $(\mathrm{mmol} / \mathrm{L})$ & $3.6(1.35)$ & $3.9(1.75)$ \\
\hline Mean (SD) creatinine $(\mathrm{g} / \mathrm{L})$ & $57.1(13.54)$ & $57.2(13.85)$ \\
\hline Median (interquartile range) proteinuria (g/24h) & $1.10(0.56-3.04)$ & $1.13(0.57-2.79)$ \\
\hline Proteinuria $\geq 3 \mathrm{~g} / 24 \mathrm{~h}$ & $23(26)$ & $20(22)$ \\
\hline Median (interquartile range) fetal weight centile on GROW chart & $11.0(1.4-37.7)$ & $13.3(2.9-33.9)$ \\
\hline \multicolumn{3}{|l|}{ Fetal weight on GROW chart: } \\
\hline «3rd centile & $28(31)$ & $24(27)$ \\
\hline 3rd to $<10$ th centile & $14(16)$ & $18(20)$ \\
\hline z10th centile & $47(53)$ & $48(53)$ \\
\hline \multicolumn{3}{|l|}{ Fetal weight on WHO charts: } \\
\hline «3rd centile & $27(30)$ & $26(29)$ \\
\hline 3 rd to $<10$ th centile & $14(16)$ & $19(21)$ \\
\hline 210th centile & $48(54)$ & $45(50)$ \\
\hline Absent end diastolic blood flow on umbilical artery Doppler velocimetry & $0(0)$ & $1(1)$ \\
\hline
\end{tabular}

data remained blinded to study drug allocation. The total length of admission in any neonatal unit was also non-significantly shorter in the metformin arm than placebo arm (18 days $v 30$ days; a difference of 12 days, geometric mean ratio $0.82,0.57$ to 1.18 ) (supplementary figure E).

\section{Adherence and adverse events}

Table 3 details the numbers of participants who discontinued or reduced their drugs. Three women in the metformin arm and five in the placebo arm stopped treatment as they were discharged or declined further admission (fig 1). Compared with women in the placebo arm, women in the metformin arm experienced more diarrhoea $(33 \% \vee 6 \%$; difference $27.3 \%$, 95\% confidence interval $16.3 \%$ to $38.2 \%$ ) and a non-significant increase in nausea (21\% v 11\%; $10.0 \%, 95 \%$ confidence interval $-0.8 \%$ to $20.8 \%$ ). No serious adverse events related to the trial drugs were identified.

\section{Discussion}

In this trial of women with a diagnosis of preterm pre-eclampsia at $<32$ weeks' (+0 days) gestation, those who received extended release metformin had a 7.6 day prolongation of gestation compared with women who received placebo. This difference was not statistically significant, possibly because of the higher variability in pregnancy prolongation compared with the Pre-eclampsia Intervention Trial. ${ }^{9}$ However, given there is biological plausibility ${ }^{11-13}$; the prespecified per protocol analyses (showing a 9.6 day prolongation in those continuing to take any dose of metformin and a 11.5 day difference in those who took the full dose), which were significant; and favourable neonatal outcomes (neonates in the metformin arm were 110 $\mathrm{g}$ heavier, spent a third less time in the tertiary care neonatal nursery at Tygerberg Hospital, and 12 days less in any neonatal nursery), we are cautiously optimistic that metformin treatment has a beneficial effect on prolongation of gestation. 

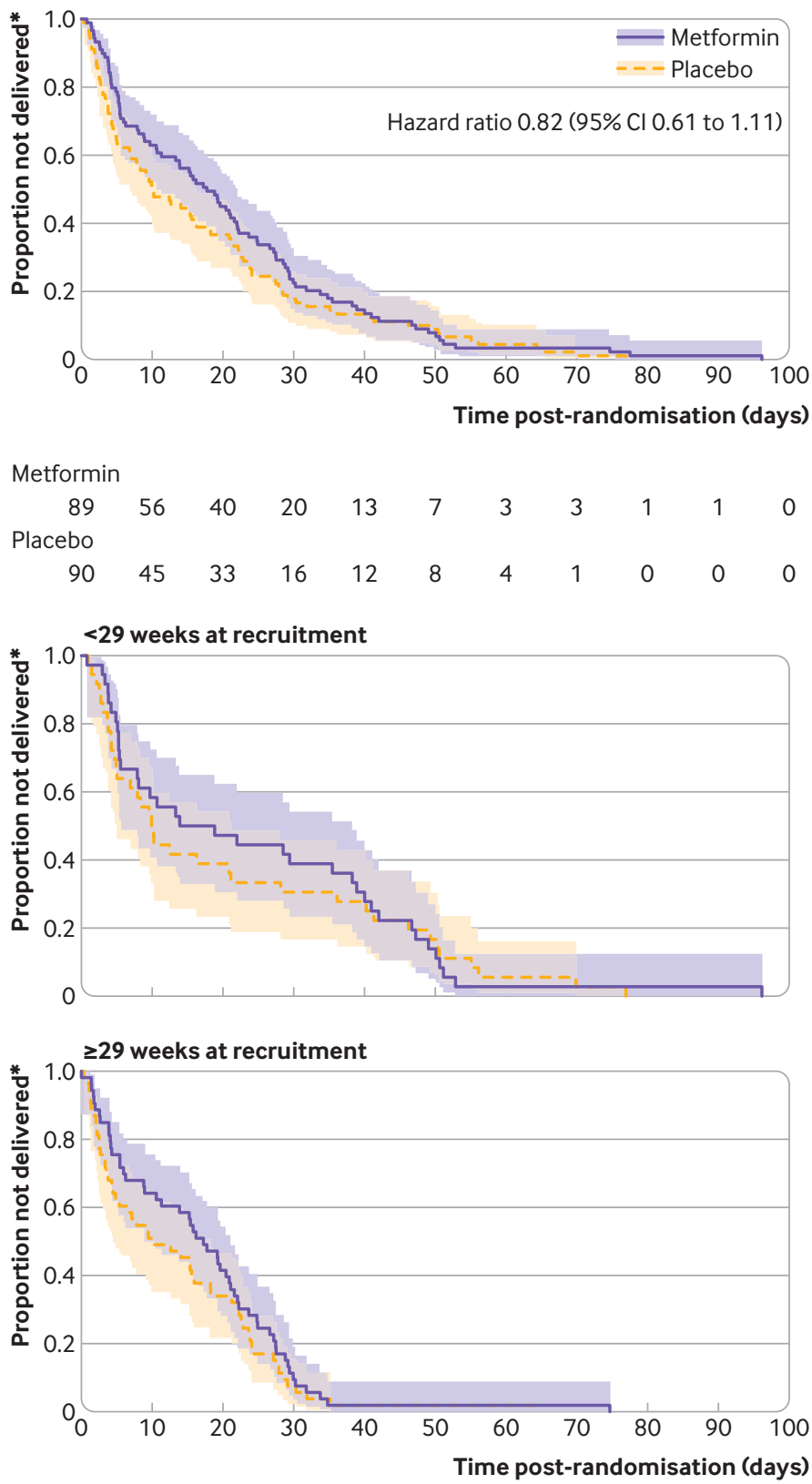

Fig 2 | Kaplan-Meier survival curves for prolongation of gestation (days) between treatment arms overall and by gestational age stratum. *Numbers indicate how many participants had not given birth at the different timepoints

Strengths and limitations of this study

The strengths of this trial are that we first performed pharmacokinetic studies in a cohort of women with preterm pre-eclampsia, a high number of those approached agreed to participate (87\%), and we were able to record detailed information on drug compliance because the women were admitted to hospital. A potential limitation is that women with preterm preeclampsia undergoing expectant management at Tygerberg Hospital deliver once they reach 34 weeks' gestation. This censored our primary outcome to this gestation. Given that $40 \%$ of women in the metformin arm and $28 \%$ in the placebo arm delivered for this indication, it is possible that prolongation of gestation among those taking metformin would have been greater had we continued expectant management beyond 34 weeks' gestation. Furthermore, this was a single centre study where the women had a high incidence of HIV, obesity, and chronic hypertension, potentially limiting the generalisability of our results. Therefore it might be worthwhile for multicentre trials to be performed in other countries to confirm whether metformin is effective in other populations and health systems. Metformin use was also associated with more gastrointestinal problems and therefore could have compromised blinding.

\section{Comparison with other studies}

Although metformin prolonged gestation, it did not reduce circulating levels of anti-angiogenic factorsnotably, soluble fms-like tyrosine kinase-1, which might be an important driver of disease pathogenesis. ${ }^{10}$ This finding suggests that metformin prolongs gestation and potentially ameliorates the underlying disease through mechanisms that do not involve a reduction in the secretion of anti-angiogenic factors. This is plausible given that preclinical studies have found that metformin might have pleiotropic actions that counter both the placental and the maternal vascular pathology of pre-eclampsia ${ }^{11-13}$ The implication is that although the reduction in soluble fms-like tyrosine kinase-1 levels remains an important conceptual approach to treating pre-eclampsia, this might not be absolutely essential to ameliorate the disease.

Although metformin is widely used to treat gestational diabetes, an advantage of repurposing the drug is that it is likely to be safe, especially if administered for a limited duration. A further advantage is that metformin is inexpensive and therefore could be widely adopted in low to middle income countries where the problem of preterm preeclampsia is most pronounced. While some concerns have been raised ${ }^{2122}$ a recent epidemiological study concluded that metformin administered during the antenatal stage did not adversely affect growth and developmental outcomes in childhood. ${ }^{23}$ Nevertheless, longer term follow-up data might be worthwhile in future trials. Gastrointestinal side effects are a disadvantage of metformin, particularly with the higher doses evaluated in our trial.

\section{Policy implications}

This trial found that metformin extended release was associated with a clinically meaningful prolongation of gestation, although the difference was not statistically significant. Further trials of metformin should be considered, such as a larger trials of women with preterm pre-eclampsia powered to confirm whether the drug can statistically significantly prolong gestation and to evaluate the benefits to neonates. Trials might also evaluate whether metformin can prevent worsening maternal organ dysfunction while birth is being planned among women with a diagnosis of late preterm pre-eclampsia, and even among those with preeclampsia at term. Lastly, it might be worth evaluating whether metformin can prevent pre-eclampsia. ${ }^{24-26}$ 


\begin{tabular}{|c|c|c|c|}
\hline Outcomes & $\begin{array}{l}\text { Metformin arm } \\
(n=89)\end{array}$ & $\begin{array}{l}\text { Placebo arm } \\
(\mathrm{n}=90)\end{array}$ & Difference* $(95 \% \mathrm{Cl})$ \\
\hline \multicolumn{4}{|l|}{ Primary outcome } \\
\hline Median (interquartile range) prolongation of gestation (days): & $17.7(5.4-29.4)$ & $10.1(3.7-24.1)$ & \multirow{3}{*}{$\begin{array}{l}1.39(0.99 \text { to } 1.95) \dagger \\
P=0.057\end{array}$} \\
\hline Minimum, maximum & $0.8,96.1$ & $0.3,76.9$ & \\
\hline Median (interquartile range) prolongation of gestation (hours): & $426(128-705)$ & $242(88-578)$ & \\
\hline Minimum, maximum & 19,2307 & 8,1846 & \\
\hline \multicolumn{4}{|l|}{ Secondary, composite, outcomes } \\
\hline Maternal‡ & $5(6)$ & $4(4)$ & $1.3(-5.2$ to 7.6$)$ \\
\hline Fetal§ & $59(66)$ & $62(70)$ & $-4.2(-17.8$ to 9.5$)$ \\
\hline Neonatalף & $14(16)$ & $16(18)$ & $-2.0(-13.0$ to 8.9$)$ \\
\hline \multicolumn{4}{|l|}{ Exploratory outcomes } \\
\hline \multicolumn{4}{|l|}{ Maternal ${ }^{\star \star}$} \\
\hline Pulmonary oedema & $4(4)$ & $2(2)$ & $2.3(-3.0$ to 7.5$)$ \\
\hline Acute renal failure (serum creatinine $\geq 125 \mu \mathrm{mol} / \mathrm{L}$ ) & $1(1)$ & $1(1)$ & $0.0(-3.1$ to 3.1$)$ \\
\hline Placental abruption & 0 & $1(1)$ & $-1.1(-3.3$ to 1.1$)$ \\
\hline Admission to $\mathrm{HCU}$ or $\mathrm{NICU}$ & $7(8)$ & $7(8)$ & $0.0(-7.8$ to 8.0$)$ \\
\hline Posterior reversible encephalopathy syndrome & 0 & $1(1)$ & $-1.1(-3.3$ to 1.1$)$ \\
\hline Left ventricular failure & 0 & $1(1)$ & $-1.1(-3.3$ to 1.1$)$ \\
\hline HELLP syndrome & $3(3)$ & $7(8)$ & $-4.4(-11.1$ to 2.3$)$ \\
\hline Platelet count $<100 \times 10^{9} / \mathrm{L}$ & $7(8)$ & $6(7)$ & $1.2(-6.4$ to 8.8$)$ \\
\hline Platelet count $<50 \times 10^{9} / \mathrm{L}$ & 0 & $1(1)$ & $-1.1(-3.3$ to 1.1$)$ \\
\hline Major postpartum haemorrhage & $2(2)$ & 0 & $2.2(-0.8$ to 5.3$)$ \\
\hline Thromboembolic disease & $1(1)$ & $1(1)$ & $0.0(-3.1$ to 3.1$)$ \\
\hline Moderate to severe ascites & $11(12)$ & $9(10)$ & $2.4(-6.9$ to 11.6$)$ \\
\hline \multicolumn{4}{|l|}{ Mean (SD) highest blood pressure during expectant management $(\mathrm{mm} \mathrm{Hg})$ : } \\
\hline Systolic & $159(12.3)$ & $157(14.2)$ & $2(-2$ to 6$)$ \\
\hline Diastolic & $95(9.4)$ & $95(9.6)$ & $0(-3$ to 3$)$ \\
\hline \multicolumn{4}{|l|}{ Mean (SD) blood pressure at delivery $(\mathrm{mm} \mathrm{Hg})$ : } \\
\hline Systolic $>160$ & $31(35)$ & $29(32)$ & $-2.6(-11.3$ to 16.6$)$ \\
\hline Diastolic $>110$ & $3(3)$ & $3(3)$ & $0.0(-5.3$ to 5.4$)$ \\
\hline \multicolumn{4}{|l|}{ Mean (SD) highest blood pressure after delivery $(\mathrm{mm} \mathrm{Hg})$ : } \\
\hline Systolic & $150(13.7)$ & $153(14.6)$ & $-3(-7$ to 2$)$ \\
\hline Diastolic & $90(10.4)$ & $91(11.3)$ & $-1(-4$ to 2$)$ \\
\hline Median (interquartile range) maternal length of stay after delivery (days) & $3(2-4)$ & $3(2-4)$ & $0.98(0.82 \text { to } 1.18)^{\star}$ \\
\hline \multicolumn{4}{|l|}{ Neonatal exploratory outcomes } \\
\hline Mean (SD birth weight $(\mathrm{g})$ & $1620(633)$ & $1510(663)$ & $110(-80$ to 300$)$ \\
\hline Mean (SD) gestation at delivery (weeks) & $32.5(2.68)$ & $31.9(2.64)$ & $0.6(-0.2$ to 1.3$)$ \\
\hline \multicolumn{4}{|l|}{ GROW centile at birth: } \\
\hline Median (interquartile range) & $2.0(0.3-12.7)$ & $1.4(0.1-12.0)$ & $0.94(0.49 \text { to } 1.79)^{\star}$ \\
\hline «3rd centile & $49(55)$ & $51(57)$ & - \\
\hline 3 rd to $<10$ th & $16(18)$ & $15(17)$ & - \\
\hline$\geq 10$ th & $24(27)$ & $24(27)$ & - \\
\hline \multicolumn{4}{|l|}{ WHO centile at birth: } \\
\hline «3rd centile & $44(49)$ & $53(59)$ & - \\
\hline 3rd to $<10$ th & $19(21)$ & $15(17)$ & - \\
\hline 210th & $26(29)$ & $22(24)$ & - \\
\hline Neonatal death & $7(8)$ & $5(6)$ & $2.3(-5.0$ to 9.6$)$ \\
\hline Grade III or IV Intraventricular haemorrhage & $2(2)$ & $4(4)$ & $-2.2(-7.5$ to 3.1$)$ \\
\hline Necrotising enterocolitis & $7(8)$ & $9(10)$ & $-2.1(-10.5$ to 6.2$)$ \\
\hline Bronchopulmonary dysplasia & 0 & 0 & - \\
\hline Apgar score $<7$ at 5 minutes & $4(4)$ & $6(7)$ & $-2.2(-8.9$ to 4.5$)$ \\
\hline Surfactant use & $12(13)$ & $15(17)$ & $-3.2(-13.7$ to 7.3$)$ \\
\hline NICU admission & $11(12)$ & $14(16)$ & $-3.2(-13.3$ to 6.9$)$ \\
\hline Intubation and mechanical ventilation & $7(8)$ & $8(9)$ & $-1.0(-9.1$ to 7.1$)$ \\
\hline Continuous positive airway pressure support & $55(62)$ & $60(67)$ & $-4.9(-18.9$ to 9.2$)$ \\
\hline Grade III or IV hyaline membrane disease & 13(15) & $13(14)$ & $0.2(-10.2$ to 10.5$)$ \\
\hline Retinopathy of prematurity & $1(1)$ & $2(2)$ & $-1.1(-4.9$ to 2.7$)$ \\
\hline Neonatal sepsis & $15(17)$ & $20(22)$ & $-5.4(-17.0$ to 6.2$)$ \\
\hline \multicolumn{4}{|l|}{ Median (interquartile range) neonatal stay (days): } \\
\hline Tertiary care nursery & $11(6.5-25.5)$ & $16(8-30)$ & $0.86(0.62 \text { to } 1.20)^{\star}$ \\
\hline Overall & $18(7-41)$ & $30(9-46)$ & $0.82(0.57 \text { to } 1.18)^{\star}$ \\
\hline \multicolumn{4}{|l|}{ Indications for delivery } \\
\hline Reached 34 weeks' gestation & $36(40)$ & $25(28)$ & $12.7(-1.1$ to 26.4$)$ \\
\hline Declined further expectant management & $1(1.1)$ & $1(1.1)$ & $0.0(-3.1$ to 3.1$)$ \\
\hline Spontaneous preterm birth & $4(4.5)$ & $1(1.1)$ & $3.4(-1.4$ to 8.2$)$ \\
\hline Maternal indication & $18(20)$ & $21(23)$ & $-3.1(-15.2$ to 9.0$)$ \\
\hline
\end{tabular}




\begin{tabular}{|c|c|c|c|}
\hline \multicolumn{4}{|l|}{ Table 2 | Continued } \\
\hline Outcomes & $\begin{array}{l}\text { Metformin arm } \\
(n=89)\end{array}$ & $\begin{array}{l}\text { Placebo arm } \\
(n=90)\end{array}$ & Difference* $(95 \% \mathrm{Cl})$ \\
\hline HELLP syndrome & $1(1.1)$ & $1(1.1)$ & $0.0(-3.1$ to 3.1$)$ \\
\hline Platelet count $<100$, no HELLP & 0 & $1(1.1)$ & $-1.1(-3.3$ to 1.1$)$ \\
\hline Imminent eclampsia & $2(2.3)$ & $2(2.2)$ & $0.0(-4.3$ to 4.4$)$ \\
\hline Loss of blood pressure control & $3(3.4)$ & $3(3.3)$ & $0.0(-5.2$ to 5.3$)$ \\
\hline Maternal ascites & $8(9)$ & $9(10)$ & $-1.0(-9.6$ to 7.6$)$ \\
\hline Pulmonary oedema & $1(1.1)$ & $1(1.1)$ & $0.0(-3.1$ to 3.1$)$ \\
\hline Renal deterioration & $3(3.4)$ & $4(4.4)$ & $-1.1(-6.7$ to 4.6$)$ \\
\hline Fetal indication & $29(33)$ & $40(44)$ & $-11.9(-26.0$ to 2.3$)$ \\
\hline Fetal distress & $29(33)$ & $38(42)$ & $-9.6(-23.7$ to 4.5$)$ \\
\hline Abnormal Doppler velocimetry result & 0 & $1(1.1)$ & $-1.1(-3.3$ to 1.1$)$ \\
\hline Poor fetal growth & 0 & $1(1.1)$ & $-1.1(-3.3$ to 1.1$)$ \\
\hline \multicolumn{4}{|c|}{ 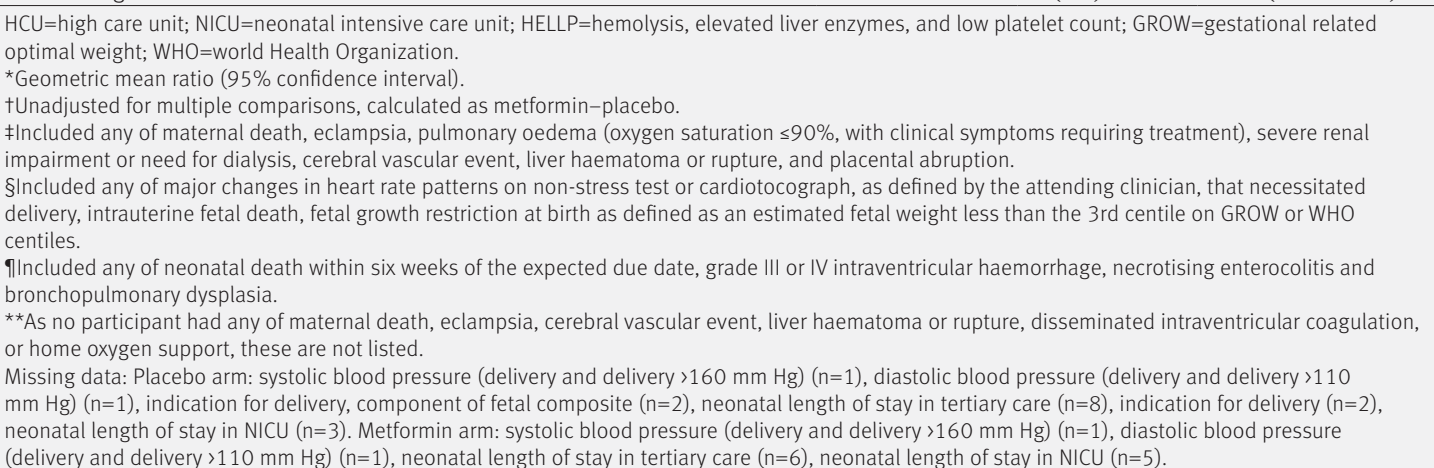 } \\
\hline
\end{tabular}

\section{Conclusions}

Based on the findings of this study, we are cautiously optimistic that extended release metformin prolongs gestation in women with preterm pre-eclampsia. Further trials are, however, needed. This trial provides

\begin{tabular}{|c|c|c|c|}
\hline & $\begin{array}{l}\text { Metformin arm } \\
(\mathrm{n}=89)\end{array}$ & $\begin{array}{l}\text { Placebo arm } \\
(n=90)\end{array}$ & $\%$ difference $(95 \% \mathrm{Cl})$ \\
\hline \multicolumn{4}{|l|}{ Side effects (days) } \\
\hline Headache: & $12(14)$ & $6(7)$ & $6.6(-2.3$ to 15.5$)$ \\
\hline 1 & $2(2)$ & $4(4)$ & - \\
\hline 2 & $3(3)$ & 0 & - \\
\hline 3 & $5(6)$ & $1(1)$ & - \\
\hline$\geq 4$ & $2(2)$ & $1(1)$ & - \\
\hline Vomiting: & $5(6)$ & $3(3)$ & $2.3(-3.9$ to 8.4$)$ \\
\hline 1 & $2(2)$ & $2(2)$ & \\
\hline 2 & $1(1)$ & 0 & \\
\hline 3 & 0 & 0 & \\
\hline$\geq 4$ & $2(2)$ & $1(1)$ & \\
\hline Nausea: & $19(21)$ & $10(11)$ & 10.0 (-0.8 to 20.8$)$ \\
\hline 1 & $4(5)$ & $5(6)$ & \\
\hline 2 & $3(3)$ & $2(2)$ & \\
\hline 3 & $6(7)$ & $1(1)$ & \\
\hline$\geq 4$ & $6(7)$ & $2(2)$ & \\
\hline Diarrhoea & $29(33)$ & $5(6)$ & 27.3 (16.3 to 38.2 ) \\
\hline 1 & $2(2)$ & $2(2)$ & \\
\hline 2 & $7(8)$ & $2(2)$ & \\
\hline 3 & $9(10)$ & $1(1)$ & \\
\hline$\geq 4$ & $11(12)$ & 0 & \\
\hline \multicolumn{4}{|l|}{ Adherence to trial drug } \\
\hline Took full treatment dose & $40(45)$ & $61(68)$ & $-22.8(-37.0$ to -8.7$)$ \\
\hline Decreased treatment dose but did not stop & $36(41)$ & $13(14)$ & $26.0(13.5$ to 38.5$)$ \\
\hline Stopped treatment & $12(13)$ & $15(17)$ & $-3.2(-13.7$ to 7.3$)$ \\
\hline Incomplete information on dosing & $1(1)$ & $1(1)$ & 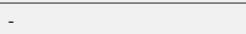 \\
\hline
\end{tabular}

Missing data: Placebo arm: headache $(n=3)$, vomiting $(n=2)$, nausea $(n=2)$, diarrhoea $(n=2)$. Metformin arm: vomiting $(n=1)$, diarrhoea $(n=1)$. proof of concept that treatment of preterm preeclampsia is possible.

We thank the members of the data monitoring and safety committee (Gerhard Theron, Adrie Bekker, and Chris Muller); staff at Tygerberg Hospital, Stellenbosch University, who identified participants; Juanita Ottaway and Liddy Griffith at the South Australian Health and Medical Research Institute for setting up and managing the offsite, online randomisation sequencing; staff from Merck Healthcare, Darmstadt, Germany; Marlien Kruger the trial pharmacist; Andrew Lytwynchuk who monitored the trial; Zukiswa Magogotya and Carlie Du Plessis our research nurses; and Ping Cannon, Tuong-Vi Nguyen, and Alesia Harper for performing the biomarker laboratory assays.

Contributors: SPW and ST (co-principal investigators and members of the trial steering committee) contributed equally to this report. CAC conceptualised the trial with SPW and ST, was the main site investigator running the trial, acquired the data, interpreted the data with RH, SPW, and ST, helped analyse the data, wrote the first draft with SPW and ST, and approved and assisted in writing the final version. She is the guarantor. RH designed the statistical plan, analysed the data, revised the work, and approved the final version. EHD performed the pharmacokinetic analysis, designed and revised the section on pharmacokinetics, and approved the final version. DRH assisted with the design of the trial and analysis of the data, revised the work, and approved the final version. SS acquired the data and samples, revised the work, and approved the final version. BWM (chair of trial steering committee) assisted with the design of the trial and analysis of the data, revised the work, and approved the final version. FB and TJK analysed the biomarkers, revised the section on biomarkers, and approved the final version. The corresponding author attests that all listed authors meet authorship criteria and that no others meeting the criteria have been omitted.

Funding: This work was supported by the Mercy Health Foundation, Peter Joseph Pappas research grant programme, Preeclampsia Foundation, and South African Medical Research Council self-initiated research grants programme. Merck Healthcare, Darmstadt, Germany donated the study drugs. The National Health and Medical Research Council of Australia provided salary support to BWM, TJK, FB, and ST. CAC received salary support from the Mercy Health Foundation. This was an investigator initiated trial. The funders had no role in the collection, analysis, interpretation of data, writing of the article, or decision to submit the article for publication.

Competing interests: All authors have completed the ICMJE uniform disclosure form at www.icmje.org/coi_disclosure.pdf and declare: support from the Mercy Health Foundation, Peter Joseph Pappas 
research grant programme, Preeclampsia Foundation, and South African Medical Research Council self-initiated research grants programme; no financial relationships with any organisations that might have an interest in the submitted work in the previous three years; no other relationships or activities that could appear to have influenced the submitted work.

Ethical approval: The trial was approved by Stellenbosch University Health Research Ethics Committee (M16/09/037), Federal Wide Assurance No 00001372 , institutional review board No IRB0005239 of Stellenbosch University.

Data sharing: The published protocol is available at https://pubmed. ncbi.nlm.nih.gov/31023758/. All relevant anonymised patient level data available on reasonable request. The protocol and statistical analysis plan and version summary can be found in supplement 2 . The dataset is available from the corresponding author.

The lead author (CAC) affirms that the manuscript is an honest, accurate, and transparent account of the study being reported; that no important aspects of the study have been omitted; and that any discrepancies from the study as originally planned (and, if relevant, registered) have been explained.

Dissemination to participants and related patient and public communities: Results of this trial will be widely distributed to clinicians and advocacy groups, including the Pre-eclampsia Foundation (https://www.preeclampsia.org/). We plan to do a press release and will post a plain language summary on our websites. We will present the findings at various meetings, including a meeting at Tygerberg Hospital, and participants who requested feedback will be contacted.

Provenance and peer review: Not commissioned; externally peer reviewed.

This is an Open Access article distributed in accordance with the Creative Commons Attribution Non Commercial (CC BY-NC 4.0) license, which permits others to distribute, remix, adapt, build upon this work non-commercially, and license their derivative works on different terms, provided the original work is properly cited and the use is noncommercial. See: http://creativecommons.org/licenses/by-nc/4.0/

1 Abalos E, Cuesta C, Grosso AL, Chou D, Say L. Global and regional estimates of preeclampsia and eclampsia: a systematic review. Eur J Obstet Gynecol Reprod Biol 2013;170:1-7. doi:10.1016/j. ejogrb.2013.05.005

2 Chappell LC, Cluver CA, Kingdom J, Tong S. Pre-eclampsia. Lancet 2021;398:341-54. doi:10.1016/S0140-6736(20)32335-7

3 Moodley J. Maternal deaths due to hypertensive disorders of pregnancy: data from the 2014-2016 Saving Mothers' Report. Obstet Gynaecol Forum 2018;28:28-32.

4 Lisonkova S, Joseph KS. Incidence of preeclampsia: risk factors and outcomes associated with early- versus late-onset disease. Am J Obstet Gynecol 2013;209:544.e1-12. doi:10.1016/j. ajog.2013.08.019

5 Lisonkova S, Sabr Y, Mayer C, Young C, Skoll A, Joseph KS. Maternal morbidity associated with early-onset and late-onse preeclampsia. Obstet Gynecol 2014;124:771-81. doi:10.1097/ AOG 0000000000000472

6 Ahmed A, Rahman M, Zhang X, et al. Induction of placental heme oxygenase- 1 is protective against TNFalpha-induced cytotoxicity and promotes vessel relaxation. Mol Med 2000;6:391-409. doi:10.1007/BF03401783

7 Paidas MJ, Tita ATN, Macones GA, et al. Prospective, randomized, double-blind, placebo-controlled evaluation of the Pharmacokinetics, Safety and Efficacy of Recombinant Antithrombin Versus Placebo in Preterm Preeclampsia. Am J Obstet Gynecol 2020;223:739.e1-13. doi:10.1016/j.ajog.2020.08.004

8 Vigil-De Gracia P, Ludmir J. Perinatal and Hemodynamic Evaluation of Sildenafil Citrate for Preeclampsia Treatment: A Randomized Controlled Trial. Obstet Gynecol 2016;128:1181-2. doi:10.1097/ AOG.0000000000001727

9 Cluver CA, Hannan NJ, van Papendorp E, et al. Esomeprazole to treat women with preterm preeclampsia: a randomized placebo controlle trial. Am J Obstet Gynecol 2018;219:388.e1-17. doi:10.1016/j. ajog.2018.07.019

10 Tong S, Kaitu'u-Lino TJ, Hastie R, Brownfoot F, Cluver C, Hannan N. Pravastatin, proton-pump inhibitors, metformin, micronutrients, and biologics: new horizons for the prevention or treatment of preeclampsia. Am J Obstet Gynecol 2020;0:S0002-9378(20)310711. doi:10.1016/j.ajog.2020.09.014

11 Brownfoot FC, Hastie R, Hannan NJ, et al. Metformin as a prevention and treatment for preeclampsia: effects on soluble fms-like tyrosine kinase 1 and soluble endoglin secretion and endothelial dysfunction. Am J Obstet Gynecol 2016;214:356.e1-15. doi:10.1016/j. ajog.2015.12.019

$12 \mathrm{Hu} \mathrm{J}$, Zhang J, Zhu B. Protective effect of metformin on a rat model of lipopolysaccharide-induced preeclampsia. Fundam Clin Pharmacol 2019;33:649-58. doi:10.1111/fcp.12501

13 Wang F, Cao G, Yi W, Li L, Cao X, Bao P. Effect of Metformin on a Preeclampsia-Like Mouse Model Induced by High-Fat Diet. Biomed Res Int 2019;2019:6547019. doi:10.1155/2019/6547019

14 Brown MA, Magee LA, Kenny LC, et al, International Society fo the Study of Hypertension in Pregnancy (ISSHP). Hypertensive disorders of pregnancy: ISSHP classification, diagnosis, and management recommendations for international practice. Vol. 72. Hypertension 2018:72:24-43 doi:10.1161/ HYPERTENSIONAHA.117.10803

15 Hall DR. Understanding expectant management of pre-eclampsia. Obstet Gynaecol Forum 2016;26:22-7.

16 Cluver C, Walker SP, Mol BW, et al. A double blind, randomised, placebo-controlled trial to evaluate the efficacy of metformin to treat preterm pre-eclampsia (PI2 Trial): study protocol. BMJ Open 2019;9:e025809. doi:10.1136/bmjopen-2018-025809

17 Harris PA, Taylor R, Thielke R, Payne J, Gonzalez N, Conde JG. Research electronic data capture (REDCap)--a metadata-driven methodology and workflow process for providing translational research informatics support. J Biomed Inform 2009;42:377-81. doi:10.1016/j. jbi.2008.08.010

18 Gardosi J, Francis A, Turner S, Williams M. Customized growth charts: rationale, validation and clinical benefits. Am J Obstet Gynecol 2018;218(2S):S609-18. doi:10.1016/j.ajog.2017.12.011

19 Kiserud T, Piaggio G Carroli G, et al. The World Health Organization Fetal Growth Charts: A Multinational Longitudinal Study of Ultrasound Biometric Measurements and Estimated Fetal Weight. PLoS Med 2017;14

20 Hall DR, Odendaal HJ, Steyn DW, Grové D. Expectant management of early onset, severe pre-eclampsia: maternal outcome. BJOG 2000;107:1252-7. doi:10.1111/j.1471-0528.2000.tb11616.x

21 Barbour LA, Scifres C, Valent AM, et al. A cautionary response to SMFM statement: pharmacological treatment of gestational diabetes. Am J Obstet Gynecol 2018;219:367.e1-7. doi:10.1016/j. ajog.2018.06.013

22 Hanem LGE, Stridsklev S, Júlíusson PB, et al. Metformin Use in PCOS Pregnancies Increases the Risk of Offspring Overweight at 4 Years of Age: Follow-Up of Two RCTs. J Clin Endocrinol Metab 2018:103:1612-21. doi:10.1210/ic.2017-02419

23 Landi SN, Radke S, Engel SM, et al. Association of Long-term Child Growth and Developmental Outcomes With Metformin vs Insulin Treatment for Gestational Diabetes. JAMA Pediatr 2019;173:160-8. doi:10.1001/jamapediatrics.2018.4214

24 Bao LX, Shi WT, Han YX. Metformin versus insulin for gestational diabetes: a systematic review and meta-analysis. / Matern Fetal Neonatal Med 2021;34:2741-53. doi:10.1080/14767058.2019.1 670804

25 Kalafat E, Sukur YE, Abdi A, Thilaganathan B, Khalil A. Metformin for prevention of hypertensive disorders of pregnancy in women with gestational diabetes or obesity: systematic review and meta-analysis of randomized trials. Ultrasound Obstet Gynecol 2018;52:706-14. doi:10.1002/uog.19084

26 Alqudah A, McKinley MC, McNally R, et al. Risk of pre-eclampsia in women taking metformin: a systematic review and meta-analysis. Diabet Med 2018;35:160-72. doi:10.1111/dme.13523

Supplementary material: additional information, figures, and tables 